\title{
Measuring the thermalization time
}

\author{
Piotr Bożek ${ }^{1,2 *}$, Iwona Wyskiel-Piekarska ${ }^{1}$ \\ ${ }^{1}$ The H. Niewodniczański Institute of Nuclear Physics, PL-31342 Kraków, Poland \\ ${ }^{2}$ Institute of Physics, Rzeszów University, PL-35959 Rzeszów, Poland
}

DOI: http://dx.doi.org/10.5689/UA-PROC-2010-09/52

\begin{abstract}
A new method of measuring the pressure anisotropy in the early stage of heavy-ion collisions is discussed. It is shown that the transverse momentum spectra, elliptic flow and interferometry radii are not sensitive to the early anisotropy. We propose the directed flow as a measure of the early transverse and longitudinal pressures. Calculations indicate that the transverse and longitudinal pressures equilibrate in less than $0.25 \mathrm{fm} / \mathrm{c}$.
\end{abstract}

\section{Introduction}

The dynamics of ultrarelativistic heavy-ion collisions can be described using the hydrodynamic model $[1,2,3]$. The fireball created in the collision consists of a dense matter close to equilibrium. The fireball expands hydrodynamically and a collective velocity field builds up. Particle production happens through the statistical emission on the freeze-out hypersurface. The spectra of emitted particles reflect the underlying collective velocity field of the fluid. In particular, the transverse momentum spectra and the elliptic flow coefficient for different particles species can be described quantitatively using hydrodynamics.

The hydrodynamic model requires that the fluid is in local thermal equilibrium. Limitations of this ideal fluid picture are discussed in terms of possible viscous corrections to the hydrodynamic equations or in terms of the limited time interval when the collective expansion takes place. Quantitative estimates show that the shear viscosity coefficient is small [4, 5] and the viscosity corrections influence significantly only the elliptic flow results. The length of the hydrodynamic stage is determined by the initial time $\tau_{0}$ needed to form the fireball. Calculations using a hard equation of state of the hot matter do not require a short initial time to describe the spectra, but to describe the femtoscopy results a short formation time $\tau_{0}=0.1-0.25 \mathrm{fm} / \mathrm{c}$ is preferred $[6,7]$. In a boost invariant geometry, observables such as the transverse momentum spectra, elliptic flow and interferometry radii reflect the accumulated transverse flow of the fluid. The longitudinal expansion sets the cooling rate of the fluid. However, during a short expansion, scenarios with different longitudinal pressures, and hence, different cooling rates, lead to similar transverse collective flows $[8,9]$.

The initial time $\tau_{0}$, when the transverse expansion starts is not equivalent to the time required for the equilibration of the system. A necessary condition for the thermalization is that the transverse and longitudinal pressures in the fluid become similar. One expects that in the initial stage of the collision, the longitudinal pressure is smaller than the transverse one.

*speaker, email: piotr.bozek@ifj.edu.pl 
Possible implementations of such a transient, nonequilibrium pressure anisotropy in hydrodynamic equations have been discussed $[10,11,12]$. However, as noted above, the presence of the pressure anisotropy in the initial stage does not change the transverse spectra, elliptic flow or femtoscopy results. It has been proposed to look at photon or dilepton emission instead $[13,14,15]$, but drawing conclusions on the thermalization rates is difficult due to unknown backgrounds. We show that the directed flow of particles is an observable extremely sensitive to the initial pressure anisotropy [16] and it can be used to estimate the thermalization time.

\section{Calculations}

The spectra of emitted particles are written as

$$
\frac{d N}{d^{2} p_{\perp} d y}=\frac{d N}{2 \pi p_{\perp} d p_{\perp} d y}\left(1+2 v_{1} \cos (\phi)+2 v_{2} \cos (2 \phi)+\ldots\right),
$$

where $v_{1}$ and $v_{2}$ are the directed and elliptic flow coefficients. In symmetric collisions the
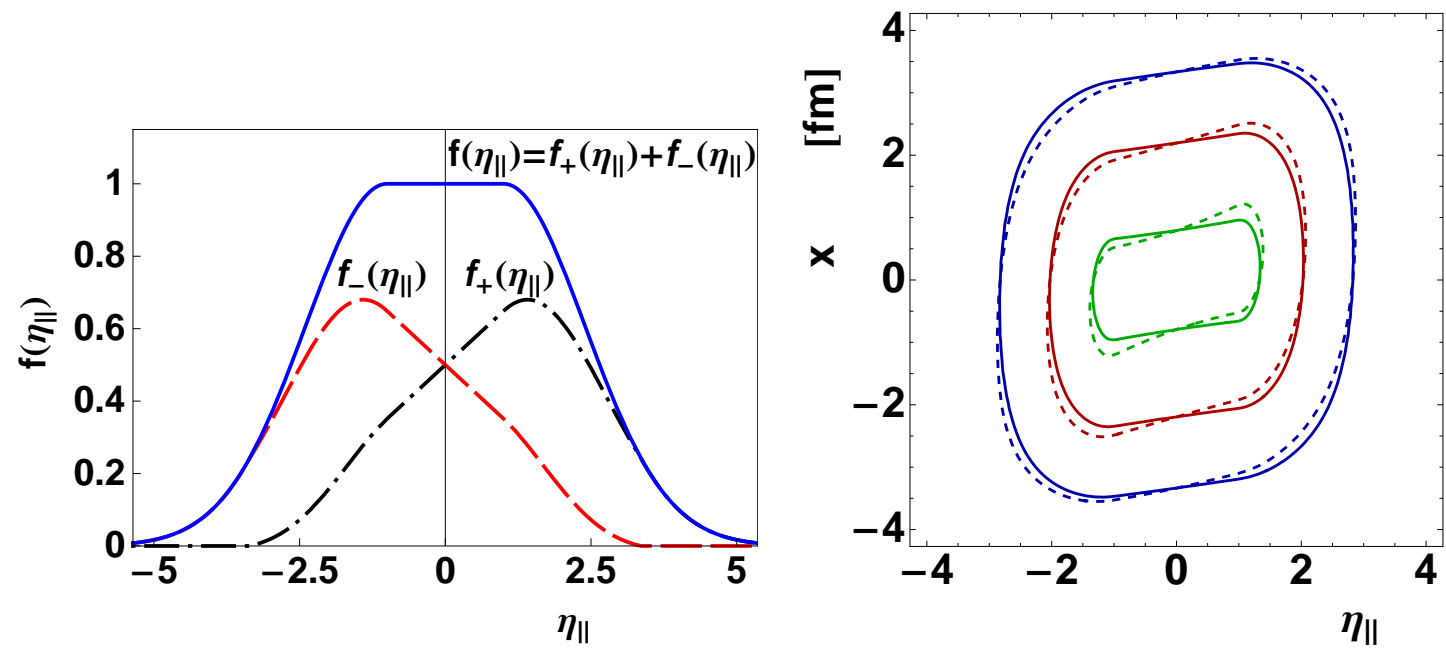

Figure 1: (left panel) The initial energy density distribution in space-time rapidity. The energy density is a sum of a two components from forward and backward going wounded nucleons [17]. (right panel) Contour plot of the initial energy density in the $\left(x-\eta_{\|}\right)$plane. The tilt of the source away from the collisions axis results from the forward (backward) peaked emission from wounded nucleons. The solid and dashed lines represent the two extreme estimates of the source tilt in the Glauber model.

directed flow coefficient $v_{1}$ is zero at central rapidities, and becomes negative (positive) at forward (backward) rapidities [18]. The formation of directed flow in ultrarelativistic collisions requires a mechanism that breaks the symmetry with respect to the collisions axis and some effective transverse and longitudinal acceleration of the fluid elements. The asymmetric emission (Fig. 1, left panel) in space-time rapidity from the forward and backward going wounded nucleons $[21,22]$ results in a tilt of the initial fireball away from the collision axis. In the right panel of Fig. 1 is shown the energy density for the tilted source. The solid and dashed 
lines represent two different source densities calculated in the Glauber model. The difference between the two distributions is a measure of the uncertainty in the initial conditions. We use the $3+1$ dimensional hydrodynamic model with a parameterization of the equation of state based on lattice QCD results [19]. The model describes the $p_{\perp}$ particle spectra and the pseudorapidity distributions for $\mathrm{Au}-\mathrm{Au}$ collisions at $\sqrt{s}=200 \mathrm{GeV}$ [20]. In the first fm/c of the hydrodynamic evolution of the tilted source, the simultaneous action of the transverse and longitudinal pressures generates a negative elliptic flow, similar as observed experimentally [17].
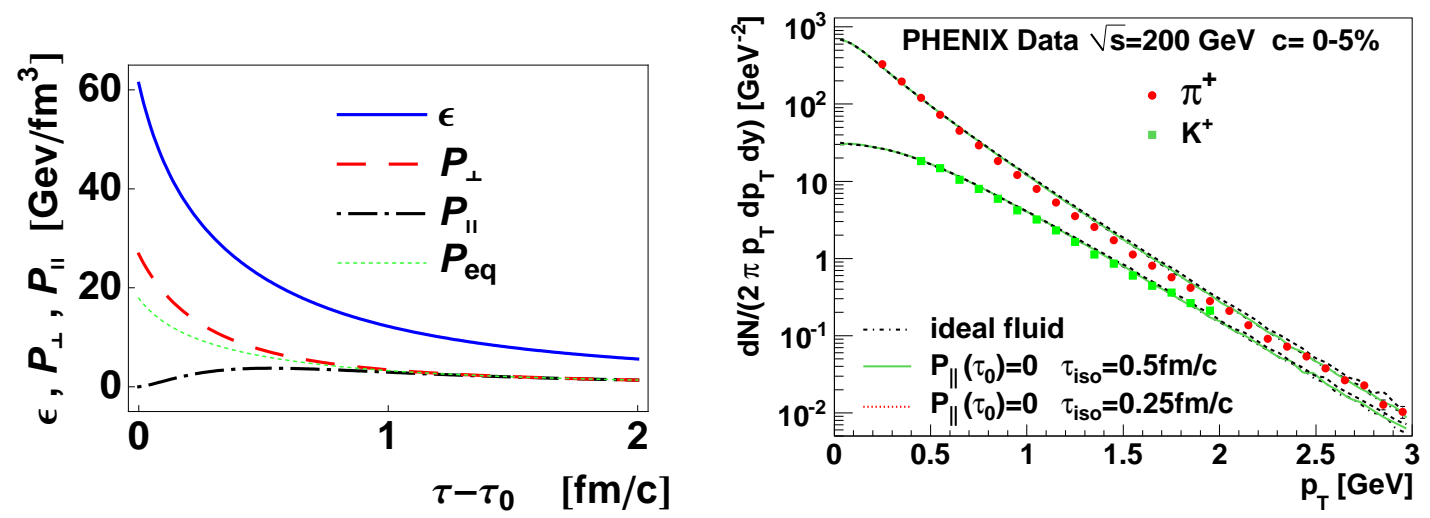

Figure 2: (left panel) Time evolution of the energy density, the longitudinal and transverse pressures at the center of the fireball, $P_{L}\left(\tau_{0}\right)=0, \tau_{i s o}=0.25 \mathrm{fm} / \mathrm{c}$. (right panel) Pion and kaon transverse momentum spectra calculated in the ideal fluid and anisotropic pressures scenarios compared to PHENIX Collaboration data [23].

We study the dynamics of the system with anisotropic pressures. In the early stage of the collisions, the energy momentum tensor in the local rest frame of the fluid is

$$
T^{\mu \nu}=\left(\begin{array}{cccc}
\epsilon & 0 & 0 & 0 \\
0 & P_{e q}+\pi / 2 & 0 & 0 \\
0 & 0 & P_{e q}+\pi / 2 & 0 \\
0 & 0 & 0 & P_{e q}-\pi
\end{array}\right) .
$$

The correction $\pi$ changes changes the transverse $P_{\perp}=P_{e q}+\pi / 2$ and the longitudinal $P_{\|}=$ $P_{e q}-\pi$ pressures. In principle, the stress correction to the pressures could be calculated from some underlying theory. The mechanisms of the pressure equilibration is still the subject of studies [26, 27, 28, 29, 30, 31]. Moreover, the models are studied in simplified geometries. In the first moments of the collisions the viscous hydrodynamics cannot be used to determine the stress correction, because the system is far from equilibrium. We are interested in the possibility of observing the occurrence of the pressure asymmetry, irrespective of its origin. We assume a simple time dependence of the correction [10]

$$
\pi(\tau)=P_{e q}\left(\tau_{0}\right) e^{\left(\tau_{0}-\tau\right) / \tau_{\text {iso }}} .
$$

It means that initially the longitudinal pressures is zero and that the pressure anisotropy decreases with a relaxation time $\tau_{\text {iso }}$ (Fig. 2). The initial density is rescaled to take into account the entropy production in the dissipative, anisotropic stage. 

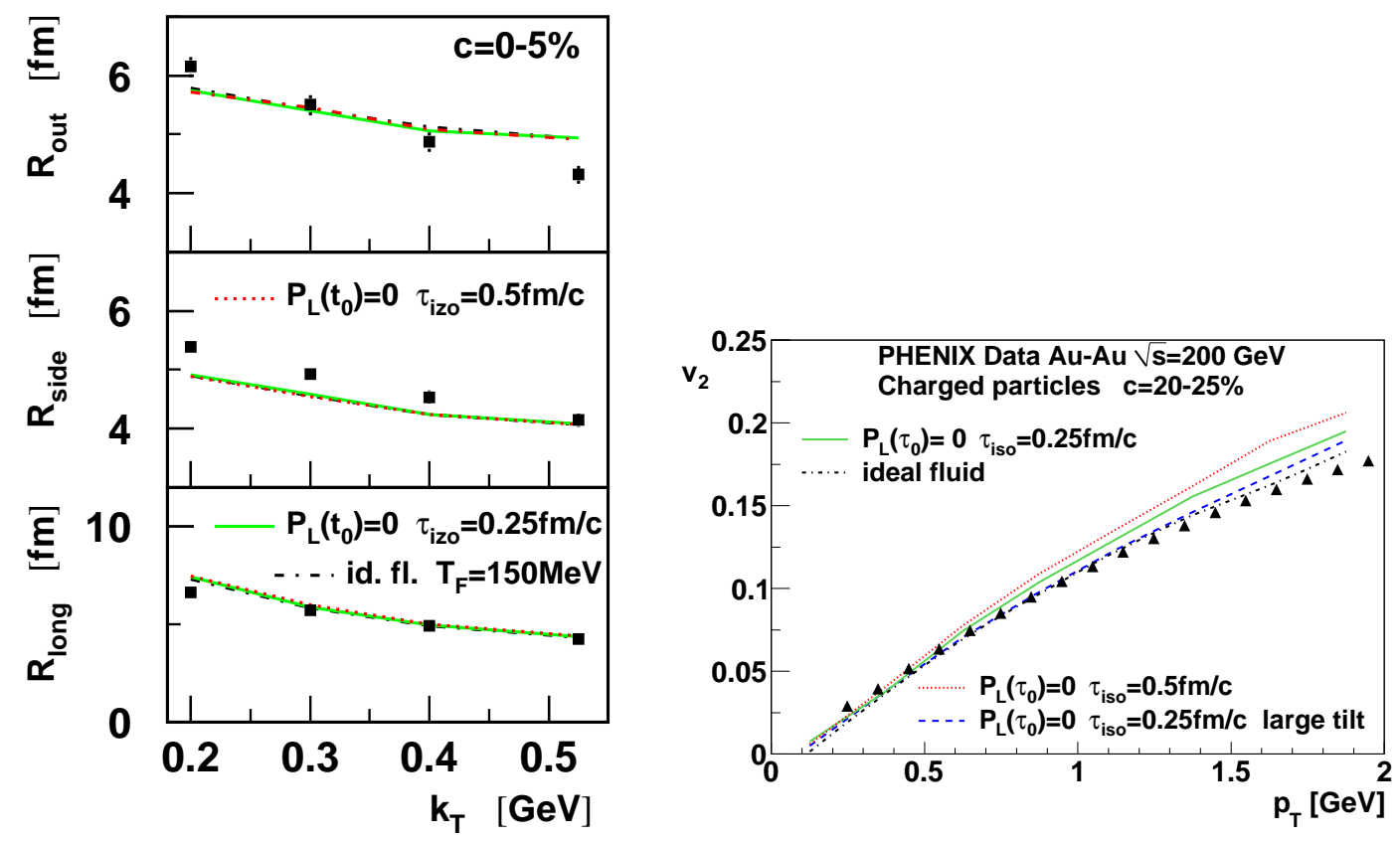

Figure 3: (left panel) Interferometry radii in central Au-Au collisions at $\sqrt{s}=200 \mathrm{GeV}$ calculated for different initial anisotropy scenarios compared to STAR Collaboration data [24]. (right panel) Elliptic flow for charged particles as function of transverse momentum for different initial anisotropy scenarios compared to PHENIX Collaboration data [25].

We simulate $\mathrm{Au}-\mathrm{Au}$ collisions at $\sqrt{s}=200 \mathrm{GeV}$ using the perfect fluid hydrodynamics $\left(\tau_{i s o}=0\right)$ and using a hydrodynamic model with the pressure anisotropy with $\tau_{\text {iso }}=0.25$ and $0.5 \mathrm{fm} / \mathrm{c}$. The transverse momentum spectra are very similar (Fig. 2). Also the interferometry radii and the elliptic flow coefficient come out almost the same in the different simulations (Fig. 3). These results reflect the universality of the transverse flow for different longitudinal pressures [9]. Moreover, these observable are determined by the form of the transverse flow at the freeze-out. The final transverse flow is built up during the whole evolution, not only in the first stage of the collisions, when the pressure anisotropy is present.

On the other hand, the directed flow is a quantity that is very sensitive to the longitudinal pressure. The acceleration of the fluid element that generates the directed flow requires the simultaneous acceleration in the transverse and longitudinal directions

$$
\begin{aligned}
& \partial_{\tau} v_{x}=-\frac{\partial_{x} P_{\perp}}{\epsilon+P}, \\
& \partial_{\tau} Y=-\frac{\partial_{\eta_{\|}} P_{\|}}{\tau(\epsilon+P)},
\end{aligned}
$$

The direction of the acceleration in the $x$ direction is usually anti-correlated with the direction of the acceleration for the fluid rapidity $Y$. It leads to a negative directed flow. Calculations show that the directed flow is formed in the first fm/c of the hydrodynamic expansion [17]. If in that time the longitudinal pressure is reduced, the final directed flow is smaller. In 

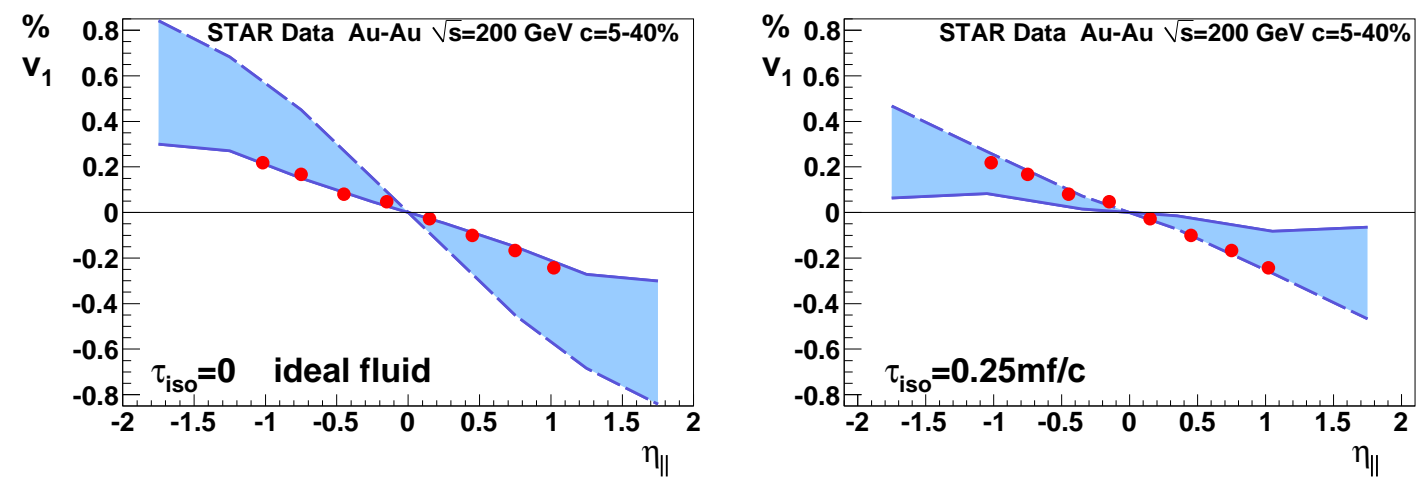

Figure 4: The directed flow calculated in $3+1$ dimensional hydrodynamics with isotropic pressure (left panel) and for the pressure anisotropy with a relaxation time $\tau_{\text {iso }}=0.25 \mathrm{fm} / \mathrm{c}$ (right panel). The shaded band represents the model uncertainty related to initial tilt of the source in the Glauber model. Data are from the STAR Collaboration for Au-Au collisions with centrality $5-40 \%$ at $\sqrt{s}=200 \mathrm{GeV}$ (dots) [18].

Fig. 4 is shown the calculated directed flow as function of pseudorapidity compared to STAR Collaboration data [18]. In the left panel the results for the perfect fluid hydrodynamics are shown. The shaded band represents the uncertainty related to the range of the initial tilt of the fireball. The results for the dynamics with asymmetric pressures are plotted in the right panel. Taking into account the uncertainty of the initial conditions we obtain the allowed limits for the thermalization (isotropization) time

$$
0 \leq \tau_{\text {iso }} \leq 0.25 \mathrm{fm} / \mathrm{c}
$$

\section{Conclusions}

We study the hydrodynamic expansion of the fireball created in a relativistic heavy-ion collision with unequal transverse and longitudinal pressures. Using a simple ansatz for the pressure anisotropy in the initial stage of the collision, we study the possibility of observing the anisotropy. We show that the transverse momentum spectra, elliptic flow and femtoscopy observables are not sensitive to this effect. Instead we propose to look at the directed flow generated in the collision. The directed flow is sensitive to the early pressure anisotropy for two reasons,

- it is formed in the first stage of the expansion,

- it is built trough a simultaneous action of the transverse and longitudinal pressures.

The directed flow is reduced if the longitudinal pressure is smaller. Comparison to experimental data sets a limit on the relaxation time for the pressure equilibration $\tau_{\text {iso }} \leq 0.25 \mathrm{fm} / \mathrm{c}$. The directed flow could serve as a sensitive constraint for microscopic models of the initial equilibration $[27,28,29,30,31,32]$. 


\section{Acknowledgments}

The work is supported by the Polish Ministry of Science and Higher Education grant No. N N202 263438.

\section{References}

[1] P. F. Kolb and U. W. Heinz, Hydrodynamic description of ultrarelativistic heavy-ion collisions, in Quark Gluon Plasma 3, edited by R. Hwa and X. N. Wang, World Scientific, Singapore, 2004, nucl-th/0305084.

[2] T. Hirano, J. Phys. G36, 064031 (2009).

[3] J.-Y. Ollitrault, (2010), arXiv: 1008.3323.

[4] P. Romatschke, Int. J. Mod. Phys. E19, 1 (2010).

[5] D. A. Teaney, (2009), arXiv: 0905.2433.

[6] W. Broniowski, M. Chojnacki, W. Florkowski, and A. Kisiel, Phys. Rev. Lett. 101, 022301 (2008).

[7] S. Pratt, Phys. Rev. Lett. 102, 232301 (2009).

[8] W. Broniowski, W. Florkowski, M. Chojnacki, and A. Kisiel, Phys. Rev. C80, 034902 (2009).

[9] J. Vredevoogd and S. Pratt, Phys. Rev. C79, 044915 (2009).

[10] P. Bożek, Acta Phys. Polon. B39, 1375 (2008).

[11] W. Florkowski and R. Ryblewski, (2010), arXiv: 1007.0130.

[12] M. Martinez and M. Strickland, Nucl. Phys. A848, 183 (2010).

[13] M. Martinez and M. Strickland, Phys. Rev. Lett. 100, 102301 (2008).

[14] B. Schenke and M. Strickland, Phys. Rev. D76, 025023 (2007).

[15] K. Dusling and S. Lin, Nucl. Phys. A809, 246 (2008).

[16] P. Bożek and I. Wyskiel-Piekarska, (2010), arXiv: 1009.0701.

[17] P. Bożek and I. Wyskiel, Phys. Rev. C81, 054902 (2010).

[18] STAR, B. I. Abelev et al., Phys. Rev. Lett. 101, 252301 (2008).

[19] M. Chojnacki and W. Florkowski, Acta Phys. Polon. B38, 3249 (2007).

[20] P. Bożek and I. Wyskiel, Phys. Rev. C79, 044916 (2009).

[21] A. Białas and W. Czyż, Acta Phys. Polon. B36, 905 (2005).

[22] A. Adil and M. Gyulassy, Phys. Rev. C72, 034907 (2005).

[23] PHEnIX, S. S. Adler et al., Phys. Rev. C69, 034909 (2004).

[24] STAR, J. Adams et al., Phys. Rev. C71, 044906 (2005).

[25] PHEnIX, A. Adare et al., Phys. Rev. Lett. 105, 062301 (2010).

[26] S. Mrowczynski, Acta Phys. Polon. B37, 427 (2006).

[27] A. Rebhan, M. Strickland, and M. Attems, Phys. Rev. D78, 045023 (2008).

[28] J. Bjoraker and R. Venugopalan, Phys. Rev. C63, 024609 (2001).

[29] Z. Xu and C. Greiner, Phys. Rev. C71, 064901 (2005).

[30] P. M. Chesler and L. G. Yaffe, Phys. Rev. D82, 026006 (2010).

[31] K. Dusling, T. Epelbaum, F. Gelis, and R. Venugopalan, (2010), arXiv: 1009.4363.

[32] B. Schenke, S. Jeon, and C. Gale, (2010), arXiv: 1009.3244. 